\title{
The method and effect of rapid establishment of a mild cognitive impairment model
}

\author{
Ting Chen ${ }^{1,2 \#}$, Qian Zhou ${ }^{1,2 \#}$, Dongjin $\mathrm{Xie}^{3}$, Chen $\mathrm{Ye}^{2}$, Linshen Huang ${ }^{1,2}$, Xianzhong Lin ${ }^{1,2}$, Lanying Lin ${ }^{1,2}$ \\ ${ }^{1}$ Department of Anesthesiology, The First Affiliated Hospital of Fujian Medical University, Fuzhou, China; ${ }^{2}$ Institute of Anesthesiology, Fujian \\ Medical University, Fuzhou, China; ${ }^{3}$ Department of Anesthesiology, The Fuzhou Second Hospital, Fuzhou, China \\ Contributions: (I) Conception and design: T Chen, X Lin, L Lin; (II) Administrative support: L Lin; (III) Provision of study materials or patients: D \\ Xie, C Ye; (IV) Collection and assembly of data: T Chen, L Huang; (V) Data analysis and interpretation: T Chen, Q Zhou; (VI) Manuscript writing: \\ All authors; (VII) Final approval of manuscript: All authors. \\ "These authors contributed equally to this work. \\ Correspondence to: Lanying Lin, MD; Xianzhong Lin, MD, PhD. Department of Anesthesiology, The First Affiliated Hospital of Fujian Medical \\ University, Fuzhou, China. Email: linlanying@163.com; lxzyjs@fjmu.edu.cn.
}

Background: To further the exploration of the pathogenesis of mild cognitive impairment (MCI), we aimed to determine the appropriate dose for a rapidly established MCI rat model using D-galactose (D-gal), with lasting cognitive effects.

Methods: In Experiment 1, we evaluated various D-gal concentrations (100-2,000 mg/kg/day), and determined that, compared with saline injections of the same volume. In Experiment 2, we evaluated the duration of the effect of $1,000 \mathrm{mg} / \mathrm{kg} /$ day D-gal injections for 1 week, with MWM testing initiated at 1 day, 1 month, and 3 months after completion of the injection regime in three model groups, respectively.

Results: In Experiment 1, D-gal injections at a concentration of 1,000 mg/kg/day for 1 week was adequate to induce a significantly worse Morris water maze (MWM) test performance and pathomorphologic changes in the hippocampus, with MWM testing initiated 1 day after completion of the injection regime. In Experiment 2, Before modeling, the overall condition (fur, mental state, foraging behavior, and activity level), body weight, swimming speed, and swimming time did not significantly differ between the control (saline injections) and model groups (D-gal injections). After modeling, MWM test performance was considerably worse (longer escape latencies and fewer platform crossings within 90 seconds) in the model groups than in the control group, without significant differences among model groups. Furthermore, movement trajectories were similar among model groups.

Conclusions: These results demonstrate that subcutaneous injections of D-gal 1,000 mg/kg/day for 1 week produce changes consistent with the characteristics and pathological processes of MCI. Thus, highdose D-gal injection allows the rapid establishment of an MCI model that is effective and sustainable.

Keywords: D-galactose (D-gal); mild cognitive impairment; animal model

Submitted Sep 22, 2020. Accepted for publication Nov 06, 2020.

doi: 10.21037/apm-20-2026

View this article at: http://dx.doi.org/10.21037/apm-20-2026

\section{Introduction}

Mild cognitive impairment (MCI) refers to a phase without any obvious clinical symptoms of dementia or objective cognitive impairment, lying between normal cognitive changes in aging and fully developed symptoms of dementia (1). MCI is a progressive neurodegenerative disease; the pathological changes in MCI are similar to those of dementia, but do not meet the criteria for dementia. Each year, approximately $10-12 \%$ of patients with MCI develop dementia (2), and more than half of patients with MCI progress to dementia within 5 years (3). 
Furthermore, accumulating clinical evidence suggests that, in elderly patients, a preoperative clinical diagnosis of possible MCI is a risk factor for severe postoperative decline in cognitive function, and even the development of Alzheimer's disease $(4,5)$. Therefore, research focusing on MCI, and in particular on its prevention and treatment, is crucial.

At present, diagnostic methods for MCI are lacking in clinical practice, and thus, clinicians rely on various neuropsychological assessment scales, such as the MiniMental State Examination and Montreal Cognitive Assessment scale (6). Animal models provide an important bridge between theoretical foundations and clinical practice. $\mathrm{D}$-galactose (D-gal) injection has been widely used in pharmacological and pathological studies to induce senescence or cognitive dysfunction in animal models owing to its simple logistics, high repeatability, minimal trauma, and comprehensive mechanism (7).

Our early study showed that subcutaneous injection of $\mathrm{D}$-gal $(1,000 \mathrm{mg} / \mathrm{kg} / \mathrm{day})$ in rats can result in mild nerve pathological changes and cholinergic system function impairment, mild oxidative damage and abated oxidation resistance, and slight decreases in spatial learning and memory abilities, with no changes in motivation and foraging behavior. Thus, D-gal injection appears to meet the major requirements of a model of the pathological processes and characteristics of MCI. However, rat behavior, biochemistry, and nerve pathological changes were only observed after dosing. Thus, it remains unclear whether these changes are sustained over a period of time after D-gal injection, further satisfying the requirements of a MCI model.

In previous studies, D-gal was injected at doses of 50 $150 \mathrm{mg} / \mathrm{kg} / \mathrm{day}$ for $6-10$ weeks, and the age of the animals ranged from 6 weeks to 6 months. So, a long period of time was required before reaching the testing cycle, with increased research costs. Therefore, we aimed to determine the appropriate dose of D-gal for a more rapidly established MCI model, and to confirm lasting cognitive impairments of this rapid model. We present the following article in accordance with the ARRIVE reporting checklist (available at http://dx.doi.org/10.21037/apm-20-2026).

\section{Methods}

\section{Animals}

A total of 40 male Sprague Dawley rats (weight, $300 \pm 50 \mathrm{~g}$,
Minhou Wu Experimental Animal Co. Ltd., China) were used in this study. All animal experiments were approved by the Ethics Committee of The First Affiliated Hospital of Fujian Medical University (Fuzhou, China), and were conducted in accordance with EU Directive 2010/63/EU for animal experiments. They were housed in standard conditions, controlled temperature $\left(22 \pm 1^{\circ} \mathrm{C}\right)$, with free access to food and water, and were kept on a 12-hour light/ dark cycle for 1 week prior to behavioral experiments. Rats with swimming and visual impairments were excluded.

\section{Experiment 1}

Twenty rats were randomly assigned to model and control groups. The rats in the model groups (Groups A, B, C, and $\mathrm{D} ; \mathrm{n}=4$ each) received hypodermic injections of $\mathrm{D}$-gal at concentrations of $100,300,1,000$, and $2,000 \mathrm{mg} / \mathrm{kg} / \mathrm{day}$, respectively, for 1 week, and the control group (Group $\mathrm{O} ; \mathrm{n}=4)$ received the same volume of saline. Rats in each group were kept in separate cages. Once a day, the rats were weighed and their overall condition, including fur, mental status, foraging behavior, and activity level, was assessed. D-gal was procured from AMRESCO Inc. (OH, USA).

\section{Experiment 2}

Twenty rats were randomly assigned to model and control groups. The rats in the model groups (Groups A, B, and C; $\mathrm{n}=5$ each) received hackles hypodermic injections of $\mathrm{D}$-gal $(1,000 \mathrm{mg} / \mathrm{kg} /$ day) for 1 week, and the control group (group $\mathrm{D} ; \mathrm{n}=5)$ received the same volume of saline. Rats in each group were kept in separate cages, and were weighed and observed as in Experiment 1.

\section{Morris water maze (MWM) testing}

The MWM apparatus consisted of a flume $(180 \mathrm{~cm}$ in diameter) with black walls, filled with water that was maintained at a temperature of $21-25^{\circ} \mathrm{C}$. An escape platform (diameter, $12 \mathrm{~cm}$ ) was placed $2 \mathrm{~cm}$ under the water and was made invisible by adding bamboo charcoal powder to the water. Software was used to accurately track and identify the rats' swimming paths. The water tank was divided into quadrants, with the escape platform located in one quadrant (the target quadrant). A quadrant was randomly selected as the starting position, and rats were placed in the water facing the tank wall. The time from entering the water to finding the escape platform 

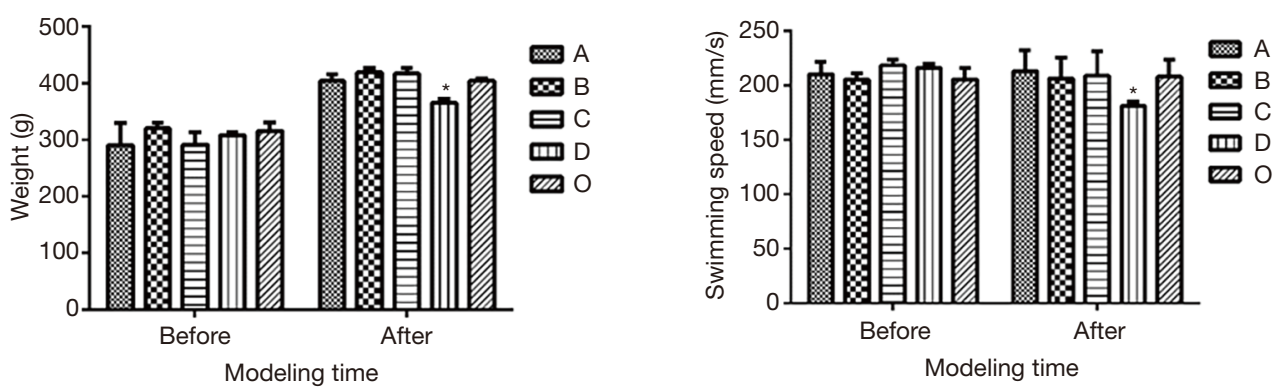

Figure 1 Experiment 1: body weight and swimming speed in the model and control groups before and after modeling. In Experiment 1, there were no significant group differences in body weight and swimming speed before modeling. After modeling, body weight and swimming speed were lower in Group D than in Group $\mathrm{O}\left({ }^{*} \mathrm{P}<0.05\right)$.

was recorded as the escape latency. When 90 seconds was reached without finding the platform, the rat was directed to the platform and allowed to remain on it for 15 seconds. All four quadrants were tested, with a training interval of 15-20 minutes over 5 consecutive days. After each testing session was completed, the rats were immediately blown dry. Following the 5-day location-based navigation test, the escape platform was removed and the rats were placed in the water in the quadrant opposite to the target quadrant (where the platform was previously located). The number of times that the rat crossed the target quadrant within 90 seconds was recorded as the number of platform crossings.

In Experiment 1, all groups started MWM testing on the day after the completion of the injection regime. In Experiment 2, Groups A and D started MWM testing the day after the completion of the injection regime, and Groups B and C started MWM testing at 1 and 3 months after the completion of the injection regime, respectively.

\section{Tissue preparation and histological assessment}

Twenty-four hours after the completion of MWM testing, the rats were anesthetized and perfused with normal saline, followed by $4 \%$ paraformaldehyde in $0.1 \mathrm{M}$ phosphate buffer ( $\mathrm{pH}, 7.4)$. On the second day, paraffin embedding was performed, and coronal sections of partial hippocampal tissues were stained with hematoxylin and eosin. Pathological changes in hippocampal tissues were observed on $10 x$ and $40 \times$ light microscopy. As MWM testing was initiated at different timepoints in Experiment 2 (i.e., at 1 day, 1 month, and 3 months after completion of the injection regime in Groups A, B, and C, respectively), histological assessment was performed at the corresponding timepoints.

\section{Statistical analysis}

Data are presented as mean \pm standard error. Data regarding body weight, number of platform crossings, swimming speed, and protein content were tested for normality and variance homogeneity. When these conditions were met, group differences were evaluated by one-way analysis of variance (ANOVA), using the least significant difference for multiple pairwise comparisons. When conditions of normality and variance homogeneity were not met, group differences were evaluated using the nonparametric ranksum test. Interactions between time (in the MWM testing procedure) and group factors were evaluated by two-way ANOVA with repeated measurements. $\mathrm{P}$ values less than 0.05 were considered statistically significant.

\section{Results}

\section{Animal characteristics}

In Experiment 1, there were no significant group differences in body weight and swimming speed prior to modeling. After modeling, body weight and swimming speed were lower in Group D than in Group O (Figure 1, $\mathrm{P}<0.05$ ). Additionally, foraging behaviors, drinking, and activity level were reduced in Group D compared to Group O. However, there were no significant differences among the other groups.

In Experiment 2, body weight, swimming speed, and swimming time did not significantly differ between Group A and Group D, and between Group B and Group C. However, the body weight was greater, and swimming speed was lower in Groups B and C compared to Group D (Figure $2, \mathrm{P}<0.05$ ). 

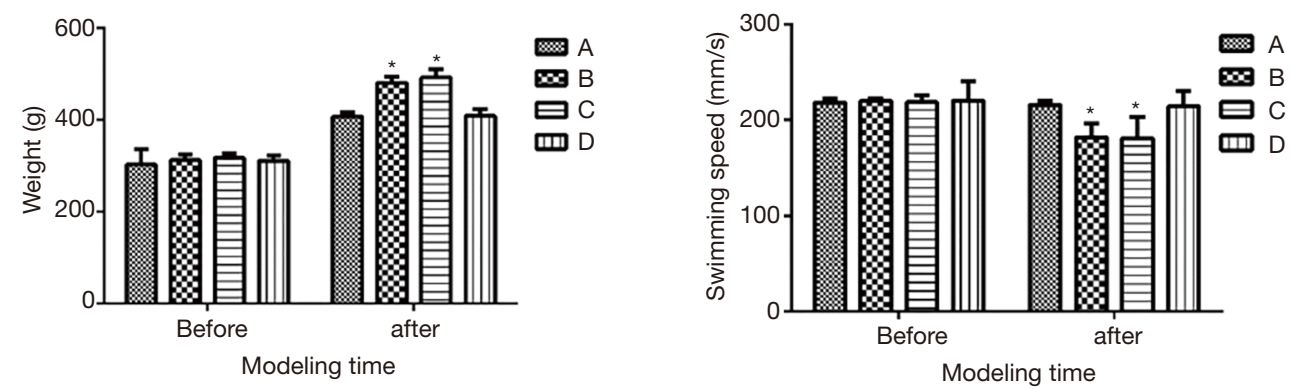

Figure 2 Experiment 2: body weight and swimming speed in the model and control groups before and after modeling. In Experiment 2, there were no significant group differences in body weight and swimming speed before modeling. However, the body weight was greater and swimming speed was lower in Groups B and C compared to Group D $\left({ }^{*} \mathrm{P}<0.05\right)$.

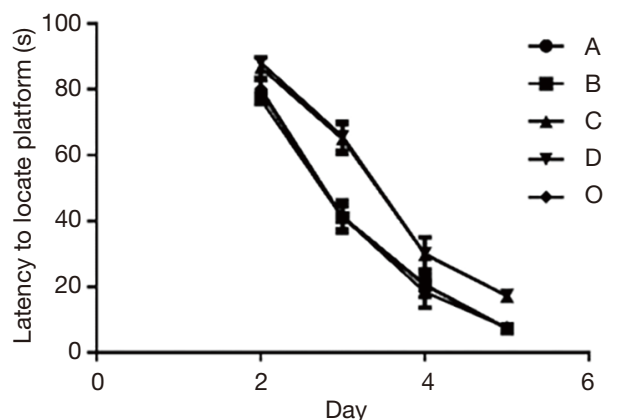

Figure 3 Experiment 1: Morris water maze (MWM) navigation test showing escape latency in the model and control groups. In Experiment 1, the MWM navigation test showed no notable differences in escape latency between Groups A and B and Group O. However, compared to Group O, Groups C and D had substantially longer escape latencies during the incubation period, from day 2 to $5(\mathrm{P}<0.05)$.

\section{MWM test performance}

In Experiment 1, the MWM navigation test showed no notable differences in escape latency between Groups A and B and Group O. However, compared to Group O, Groups $\mathrm{C}$ and $\mathrm{D}$ had substantially longer escape latencies during the incubation period, from day 2 to 5 (Figure 3, $\mathrm{P}<0.05$ ). Similarly, the MWM space exploration test showed no significant differences in the number of platform crossings between Group O and Groups A and B. In contrast, the number of platform crossings was markedly lower in Groups $\mathrm{C}$ and D than in Group $\mathrm{O}$ (Figure 4, $\mathrm{P}<0.05$ ), suggesting that Groups $\mathrm{C}$ and $\mathrm{D}$ had impaired spatial working memory.

In Experiment 2, the MWM navigation test showed

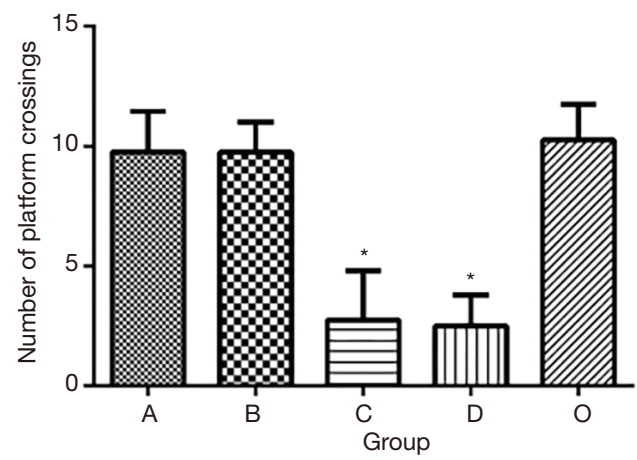

Figure 4 Experiment 1 the Morris water maze (MWM) space exploration test showing the number of platform crossings in the model and control groups. The MWM space exploration test showed no significant differences in the number of platform crossings between Group $\mathrm{O}$ and Groups $\mathrm{A}$ and B. In contrast, the number of platform crossings was markedly lower in Groups $\mathrm{C}$ and $\mathrm{D}$ than in Group $\mathrm{O}\left({ }^{*} \mathrm{P}<0.05\right)$.

that the escape latency was substantially longer in Group A than in Group D during the incubation period, from day 2 to 5 (Figure $5, \mathrm{P}<0.05$ ). However, there were no significant differences in escape latency among Groups A, B, and C. Similarly, the MWM space exploration test showed no notable differences in the number of platform crossings among Groups A, B, and C, while the number of platform crossings was markedly lower in Group A than in Group D (Figure 6, $\mathrm{P}<0.05$ ).

\section{Histological assessments}

In Experiment 1, there were no obvious neuronal changes in the CA1 region of the hippocampus in Groups A, B, and 


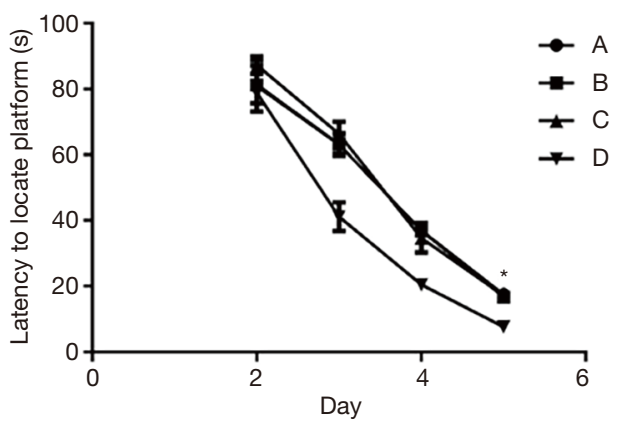

Figure 5 Experiment 2: Morris water maze (MWM) navigation test showing escape latency in the model and control groups. In Experiment 2, the MWM navigation test showed that the escape latency was substantially longer in Group A than in Group D during the incubation period, from day 2 to $5\left({ }^{*} \mathrm{P}<0.05\right)$.

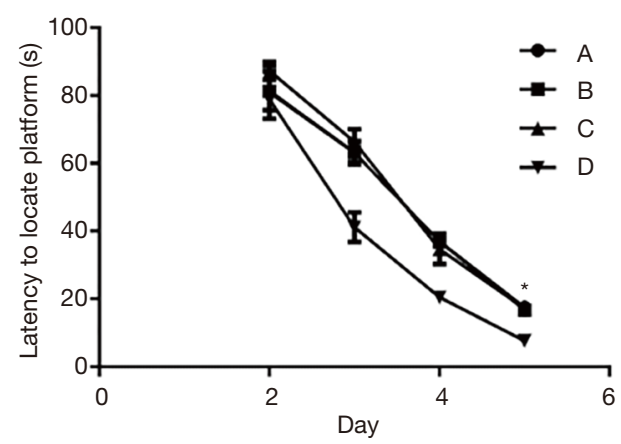

Figure 6 Experiment 2: the Morris water maze (MWM) space exploration test showing the number of platform crossings in the model and control groups. There were no notable differences in escape latency among Groups A, B, and C. Similarly, the MWM space exploration test showed no significant differences in the number of platform crossings among Groups A, B, and C, while the number of platform crossings was considerably lower in Group A than in Group D $\left({ }^{*} \mathrm{P}<0.05\right)$.

O. However, neuronal loss was observed in Groups C and D, with mild vertebral apoptosis, disarrangement, and density reduction, and some cells showed cytoplasmic condensation, deep staining, and even apoptosis. These changes were more serious in Group D than in Group C (Figure 7).

In Experiment 2, there were no obvious neuronal changes in the CA1 region of the hippocampus in Group D. However, neuronal loss was observed in Groups A, $\mathrm{B}$, and $\mathrm{C}$, with mild vertebral apoptosis, disarrangement, and density reduction, and some cells showed cytoplasmic condensation, deep staining, and even apoptosis. However, pairwise comparisons showed no significant differences in pathological neuronal changes among Groups A, B, and C (Figure 8). Thus, short-term D-gal injections can cause lasting mild pathological changes in the CA1 region of the hippocampus.

\section{Discussion}

An animal model of MCI must reflect the characteristics of aging, mild memory impairment, mild neuropathy, changes in the cholinergic system, changes in cerebral blood vessels, as well as normal and foraging behavior. Pepeu stated that an animal model established to verify the potential efficacy of a drug does not need to be overly complicated, but it should be easily applied in large quantities and reasonably priced (8). The current methods for the establishment of cognitive dysfunction in rats mainly involve permanent ligation of bilateral common carotid arteries (9), subcutaneous D-gal injection (10), and subcutaneous or intraperitoneal injection of organophosphorus or scopolin $(11,12)$. However, bilateral common carotid artery ligation causes severe cerebral ischemia in a short amount of time; it is difficult for the cerebral blood supply and oxygen supply to compensate rapidly, resulting in high animal mortality and increased research costs (13). Furthermore, organophosphorus injection easily leads to toxic hepatitis, liver cell degeneration, and necrosis due to the biological transformation of organophosphorus in the liver, which results in the release of tumor necrosis factor and other inflammatory mediators (14). Additionally, scopine hydrochloride injection primarily manifests as damage to the cholinergic system, which is not adequately comprehensive for the establishment of a model of cognitive dysfunction.

In contrast, D-gal injection can induce senescence or cognitive dysfunction in animal models comprehensively and easily, with high repeatability and minimal trauma. $\mathrm{D}$-gal is metabolized into glucose at normal physiological concentrations, and is converted into aldose and hydroperoxide at high doses, producing superoxide anions and reactive oxygen species (ROS) via the galactose-oxidase reaction. Accumulation of advanced glycation end-products (AGEs) results from its reactions with free amines (15). D-gal produces its neurotoxic effects by forming ROS and AGEs. Research has shown that D-gal can accelerate caspase-mediated neuronal apoptosis and inhibit neuronal formation. Injection of a large dose of D-gal in animals results in increased ROS products, reduced antioxidant 

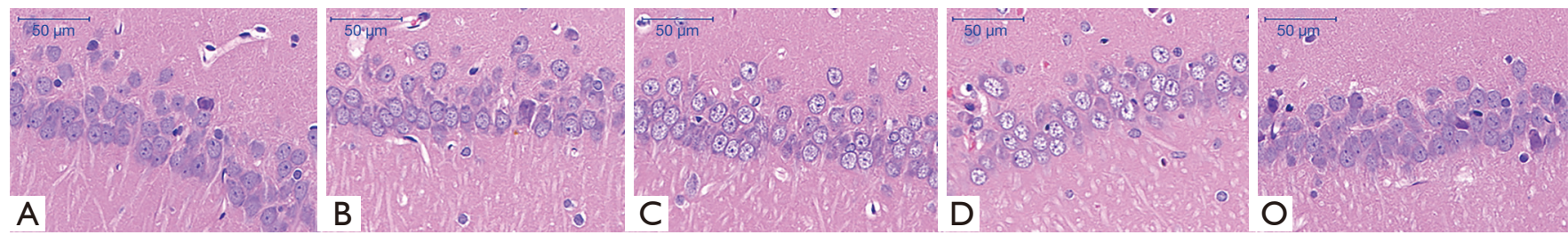

Figure 7 Experiment 1: hematoxylin and eosin stains showing neuronal changes in the CA1 region of the hippocampus. In Experiment 1, there were no obvious neuronal changes in the CA1 region of the hippocampus in Groups A, B, and O. However, neuronal loss was observed in Groups C and D, with mild vertebral apoptosis, disarrangement, and density reduction, and some cells showed cytoplasmic condensation, deep staining, and even apoptosis. These changes were more serious in Group D than in Group C. Scale bar =50 $\mu \mathrm{m}$.
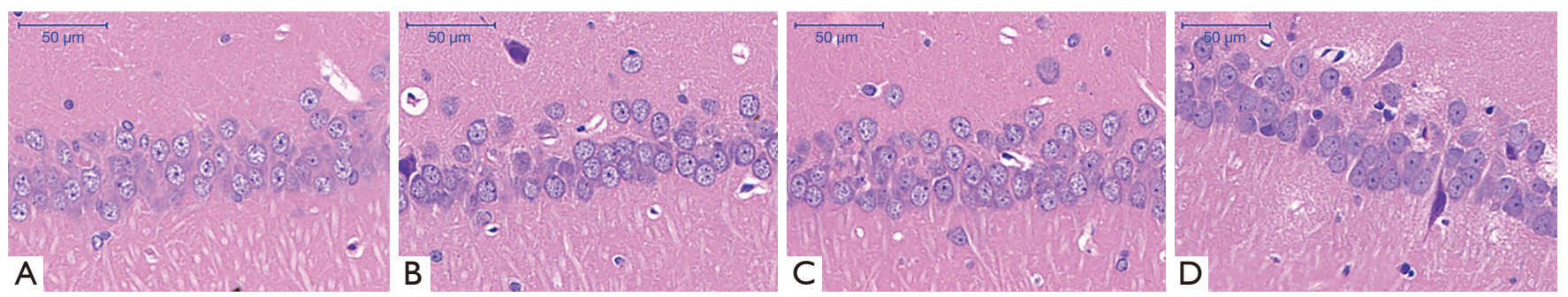

Figure 8 Experiment 2: hematoxylin and eosin stains showing neuronal changes in the CA1 region of the hippocampus. In Experiment 2, there were no obvious neuronal changes in the CA1 region of the hippocampus in Group D. However, neuronal loss was observed in Groups A, B, and C, with mild vertebral apoptosis, disarrangement, and density reduction, and some cells showed cytoplasmic condensation, deep staining, and even apoptosis. However, pairwise comparisons showed no significant differences in pathological neuronal changes among Groups A, B, and C. Scale bar =50 $\mu \mathrm{m}$.

enzyme activity in the brain, accumulation of AGEs, and subsequent hippocampal neuronal injury. This triggers apoptosis, increased brain- and liver-tissue malondialdehyde (MDA), and decreased super oxidase dismutase (SOD) activity. MDA is the main and most studied product of polyunsaturated fatty acid peroxidation, which is often used as a marker of oxidative stress; MDA content represents the level of membrane lipid peroxidation in vivo. SODs are a family of enzymes that catalyze the dismutation of peroxide free radicals; SOD activity directly reflects the antioxidant capacity of the body.

A major drawback of previous studies on D-gal injection as a model of MCI is the length of time required to establish the model. In these studies, the D-gal dose was 50$150 \mathrm{mg} / \mathrm{kg} /$ day and the injection time was usually $6-10$ weeks. However, Haider et al. showed that subacute injection of high-dose D-gal (300 mg/kg/day) could induce significant neurobehavioral and neurochemical disorders as early as 1 week (16). Therefore, in the present study, we evaluated the effects of subcutaneous D-gal injections on cognitive function at a range of concentrations (100,
$300,1,000$, and $2,000 \mathrm{mg} / \mathrm{kg} /$ day) for 1 week in order to determine the appropriate dose of D-gal for a more rapidly established MCI rat model.

Both Experiment 1 and 2 in the present study showed that large-dose D-gal injection leads to degenerative changes in hippocampal neurons and the loss of some vertebral cells in the CA1 hippocampal area, with cytoplasmic shrinkage, hyperchromia, and apoptosis in vertebral cells, as well as progressive pathological changes. Furthermore, the present study established a manufacturing method for a relatively stable MCI animal model. This method can conveniently, effectively, and efficiently cause mild memory impairment and pathological damage to the CA1 hippocampal area in Sprague Dawley rats, without excessive damage to brain tissue structure and function. Thus, this D-gal rapid modeling method has good validity and repeatability.

\section{Conclusions}

The innovations established by the present study can 
be summarized as follows: (I) an MCI rat model can be rapidly established by subcutaneous injection of D-gal $1,000 \mathrm{mg} / \mathrm{kg} / \mathrm{day}$ for 1 week; and (II) behavioral and neuropathological changes are observed at 1 and 3 months after the establishment of the model. Thus, the characteristics of the MCI model established by subcutaneous injection of $\mathrm{D}$-gal at this dose offer obvious feasibility and viability.

In recent years, the aging population in China and worldwide has become increasingly prominent. According to World Health Organization statistics, the elderly population (over 65 years old) in China will reach 200 million people by 2020 . This is accompanied by an increase in the incidence of cognitive dysfunction in elderly patients. Therefore, we hope that our rapidly established MCI rat model enables further scientific research regarding the potential mechanisms in the occurrence and development of MCI from the perspective of an animal model, so as to better serve clinical practice.

\section{Acknowledgments}

Funding: This work was supported by the Natural Science Foundation of Fujian Province, China (Grant No. 2018J01178) and the Foundation of Sailing, China (Grant No. 2019QH1081).

\section{Footnote}

Reporting Checklist: The authors have completed the ARRIVE reporting checklist. Available at http://dx.doi. org/10.21037/apm-20-2026

Data Sharing Statement: Available at http://dx.doi. org/10.21037/apm-20-2026

Conflicts of Interest: All authors have completed the ICMJE uniform disclosure form (available at http://dx.doi. org/10.21037/apm-20-2026). The authors have no conflicts of interest to declare.

Ethical Statement: The authors are accountable for all aspects of the work in ensuring that questions related to the accuracy or integrity of any part of the work are appropriately investigated and resolved. All animal experiments were approved by the Ethics Committee of The First Affiliated Hospital of Fujian Medical University (Fuzhou, China), and were conducted in accordance with
EU Directive 2010/63/EU for animal experiments.

Open Access Statement: This is an Open Access article distributed in accordance with the Creative Commons Attribution-NonCommercial-NoDerivs 4.0 International License (CC BY-NC-ND 4.0), which permits the noncommercial replication and distribution of the article with the strict proviso that no changes or edits are made and the original work is properly cited (including links to both the formal publication through the relevant DOI and the license). See: https://creativecommons.org/licenses/by-nc-nd/4.0/.

\section{References}

1. Petersen RC. Mild cognitive impairment as a diagnostic entity. J Intern Med 2004;256:183-94.

2. Petersen RC, Roberts RO, Knopman DS, et al. Mild cognitive impairment: ten years later. Arch Neurol 2009;66:1447-55.

3. Petersen RC. Mild cognitive impairment as a diagnostic entity. J Intern Med 2004;256:183-94.

4. Kazmierski J, Banys A, Latek J, et al. Mild cognitive impairment with associated inflammatory and cortisol alterations as independent risk factor for postoperative delirium. Dement Geriatr Cogn Disord 2014;38:65-78.

5. Hudetz JA, Patterson KM, Pagel PS. Comparison of preexisting cognitive impairment, amnesic mild cognitive impairment, and multiple domain mild cognitive impairment in men scheduled for coronary artery surgery. Eur J Anaesthesiol 2012;29:320-5.

6. Ciesielska N, Sokołowski R, Mazur E, et al. Is the Montreal Cognitive Assessment (MoCA) test better suited than the Mini-Mental State Examination (MMSE) in mild cognitive impairment (MCI) detection among people aged over 60? Meta-analysis. Psychiatr Pol 2016;50:1039-52.

7. Xu Y, Li Y, Ma L, et al. d-galactose induces premature senescence of lens epithelial cells by disturbing autophagy flux and mitochondrial functions. Toxicol Lett 2018;289:99-106.

8. Pepeu G. Mild cognitive impairment: animal models. Dialogues Clin Neurosci 2004;6:369-77.

9. Mansour A, Niizuma K, Rashad S, et al. A refined model of chronic cerebral hypoperfusion resulting in cognitive impairment and a low mortality rate in rats. J Neurosurg 2018;131:892-902.

10. Chiroma SM, Mohd Moklas MA, Mat Taib CN, et al. $\mathrm{d}$-galactose and aluminium chloride induced rat model with cognitive impairments. Biomed Pharmacother 
2018;103:1602-8.

11. Flannery BM, Bruun DA, Rowland DJ, et al. Persistent neuroinflammation and cognitive impairment in a rat model of acute diisopropylfluorophosphate intoxication. J Neuroinflammation 2016;13:267.

12. Malik J, Karan M, Vasisht K. Attenuating effect of bioactive coumarins from Convolvulus pluricaulis on scopolamine-induced amnesia in mice. Nat Prod Res 2016;30:578-82.

13. Huang WG, Guo FF, Liu WH, et al. Improvement of vascular dementia model in rats. Chin J Comparat Med 2011;21:50-2.

14. Ouyang YH, Li SL, Song W, et al. Expression of SOCS-
3, TNF-ain liver and spleen of mice induced by acute organophosphorus pestidde poisoning. Chin J Emerg Med 2009;18:175-9.

15. Wu DM, Lu J, Zheng YL, et al. Purple sweet potato color repairs d-galactose-induced spatial learning and memory impairment by regulating the expression of synaptic proteins. Neurobiol Learn Mem 2008;90:19-27.

16. Haider S, Liaquat L, Shahzad S, et al. A high dose of short term exogenous $\mathrm{D}$-galactose administration in young male rats produces symptoms simulating the natural aging process. Life Sci 2015;124:110-9.

(English Language Editor: A. Kassem)

Cite this article as: Chen T, Zhou Q, Xie D, Ye C, Huang L, Lin X, Lin L. The method and effect of rapid establishment of a mild cognitive impairment model. Ann Palliat Med 2020;9(6):41194126. doi: 10.21037/apm-20-2026 